\title{
Sensibilidad al benzonidazol de cepas de Trypanosoma cruzi sugiere la circulación de cepas naturalmente resistentes en Colombia
}

\author{
Ana María Mejía-Jaramillo¹, Geysson Javier Fernández¹, Marleny Montilla², \\ Rubén Santiago Nicholls², Omar Triana-Chávez ${ }^{1}$

\footnotetext{
1 Grupo Biología y Control de Enfermedades Infecciosas, Universidad de Antioquia, Medellín, Colombia

2 Grupo de Parasitología, Instituto Nacional de Salud, Bogotá, D.C., Colombia
}

\begin{abstract}
Introducción. La enfermedad de Chagas, causada por Trypanosoma cruzi, es uno de los problemas más graves de salud pública en el continente americano. El benzonidazol es uno de los dos medicamentos utilizados para tratar la enfermedad de Chagas. Sin embargo, la variación de la sensibilidad del parásito a este medicamento es una de las principales causas del fracaso del tratamiento.

Objetivo. Evaluar la sensibilidad in vitro al benzonidazol de cepas colombianas de T. cruzi de diferentes orígenes y procedencia geográfica.

Materiales y métodos. Treinta y tres cepas colombianas de T. cruzi aisladas de humanos, vectores y mamíferos, se analizaron in vitro mediante el micrométodo enzimático de MTT para determinar la concentración inhibitoria $50\left(\mathrm{Cl}_{50}\right)$ al benzonidazol. Se estudió la correlación entre la sensibilidad in vitro al medicamento y diferentes parámetros biológicos y eco-epidemiológicos.

Resultados. El análisis de sensibilidad al medicamento indicó que el $36 \%$ de las cepas eran sensibles, el $48 \%$, parcialmente resistentes y, el $16 \%$, resistentes al benzonidazol. Los análisis de correlación entre las $\mathrm{Cl}_{50}$ con algunos parámetros biológicos y eco-epidemiológicos, mostraron diferencias en cuanto a la sensibilidad según el origen biológico y el área geográfica de procedencia de la cepa.

Conclusiones. Existe una gran variabilidad en cuanto a la sensibilidad al benzonidazol de las cepas circulantes de T. cruzi en Colombia, lo cual sugiere la presencia de cepas naturalmente resistentes en el país.
\end{abstract}

Palabras clave: Trypanosoma cruzi, enfermedad de Chagas/terapia, inmunidad innata, Colombia.

\section{Trypanosoma cruzi strains resistant to benznidazole occurring in Colombia}

Introduction. Chagas disease caused by Trypanosoma cruzi is one of the most serious public health problems in the Americas. Benznidazole is one of two drugs used to treat Chagas' disease. However, the variation in susceptibility of the parasite to this drug is one of the main causes of treatment failure.

Objective. The in vitro susceptibility to benznidazole was assessed in Colombian strains of $T$. cruzi from several sources and geographical regions.

Materials and methods. Thirty-three Colombian T. cruzi strains were isolated from humans, vectors and mammals. These were analyzed in vitro by the MTT enzymatic micromethod to determine the $\mathrm{IC}_{50}$ to benznidazole. Additionally, the in vitro susceptibility was correlated with several biological and ecoepidemiological parameters.

Results. Thirty-six percent of the strains were considered to be sensitive, $48 \%$ partially resistant, and $16 \%$ were resistant. Correlations between the $\mathrm{IC}_{50}$ and several biological and eco-epidemiological parameters indicated that differences in susceptibility depended on the biological source and geographical origin of the strain.

Conclusions. A high degree of variability exists in the susceptibility to benznidazole of $T$. cruzi strains in Colombia. The distribution data indicate the presence and circulation of naturally resistant strains.

Key words: Trypanosoma cruzi, Chagas disease/therapy; immunity, innate; Colombia.

\footnotetext{
Contribución de los autores:

Ana María Mejía-Jaramillo y Geysson Javier Fernández contribuyeron de igual manera en este trabajo.

Ana María Mejía-Jaramillo y Omar Triana-Chávez diseñaron los experimentos, analizaron los resultados y escribieron el artículo. Geysson Javier Fernández hizo los ensayos de MTT para algunas cepas, analizó los resultados y revisó la versión final del manuscrito.

Marleny Montilla y Rubén Santiago Nicholls aislaron algunas cepas de humanos y revisaron la versión final del manuscrito.
} 
La enfermedad de Chagas, causada por el parásito Trypanosoma cruzi, afecta, al menos, 7'694.500 personas de diferentes regiones del continente americano, con, aproximadamente, 70 millones en riesgo de contraer la infección y causa cerca de 45.000 muertes anuales $(1,2)$. En Colombia, se estima que cerca del $5 \%$ de la población se encuentra infectada con el parásito y, aproximadamente, el $11 \%$ está en riesgo de contraer la infección $(3,4)$.

En la actualidad no existe vacuna para prevenir la infección y la quimioterapia está restringida a dos compuestos nitroheterocíclicos: el benzonidazol (N-benzil-2-nitroimidazol acetamida) y el nifurtimox ([3-metil-4-(nitrofurfurilidenamina)-tetrahidro-4H1,4-tiazina-1,1-dióxido]). Sin embargo, la efectividad del tratamiento puede verse afectada por algunos factores, tales como: 1) fase de la enfermedad, con $70 \%$ de los pacientes considerados recuperados en la fase aguda y sólo $20 \%$ en la fase crónica; 2) estado fisiológico, inmunológico y edad de los pacientes; 3 ) efectos colaterales de los medicamentos; 4) falta de cumplimiento del tratamiento, y 5) la expresión de resistencia a estos compuestos por parte del parásito (5-8).

La resistencia puede clasificarse en dos tipos, según los mecanismos mediante los cuales se expresa o surge: la intrínseca (natural o de novo), relacionada con la capacidad natural de los organismos para resistir a la quimioterapia inicial, y la adquirida, que se presenta cuando un organismo es inicialmente sensible a un medicamento y luego se vuelve moderado o fuertemente resistente al tratamiento por presiones con el compuesto (9-11).

Para T. cruzi se han descrito estos dos tipos de resistencia, lo que representa un gran problema de salud pública, puesto que la resistencia a medicamentos es uno de los principales obstáculos para la efectividad de los tratamientos, prevención y erradicación de las enfermedades parasitarias humanas. Algunos trabajos reportan que $27 \%$ de las cepas de $T$. cruzi aisladas de diferentes orígenes biológicos y geográficos, poseen resistencia natural al benzonidazol y al nifurtimox,

\footnotetext{
Correspondencia:

Ana María Mejía-Jaramillo, Grupo Biología y Control de Enfermedades Infecciosas, laboratorio 620, Sede de Investigación Universitaria, Universidad de Antioquia, Calle 62 №52-59, Medellín, Colombia

Teléfono: (574) 2196520

anamejia25@gmail.com
}

Recibido: 26/09/11; aceptado:03/01/12 con porcentajes de curación menores de $50 \%$ en ratones, a pesar de nunca haber estado en contacto con estos medicamentos (8). Además, se reportó que es posible inducir resistencia en este parásito, tanto in vitro como in vivo, por presiones continuas con estos medicamentos (12-15), lo cual genera poblaciones resistentes a concentraciones de benzonidazol de hasta $220 \mu \mathrm{M}$ (15), y se han propuesto algunos genes involucrados en el surgimiento del fenotipo de resistencia a este medicamento $(12,13,16-21)$. A la fecha, no se han podido correlacionar los mecanismos moleculares involucrados entre estos dos tipos de resistencia (16-21), ni tampoco la resistencia con otros parámetros biológicos, genéticos o bioquímicos de T. cruzi $(22,23)$.

En Colombia, el benzonidazol es uno de los tratamientos aprobados desde 2002 para tratar los pacientes infectados con T. cruzi $(24,25)$. Aunque, son pocos los estudios llevados a cabo para determinar la efectividad del tratamiento en pacientes colombianos, en el 2005, Guhl, et al., evaluaron la efectividad del tratamiento en escolares de 27 veredas, de tres municipios del departamento de Boyacá, y encontraron que existía una rápida conversión serológica a negativa en $75 \%$ de los pacientes, en contraste con los bajos porcentajes de curación reportados en otras partes del continente (24). En este sentido, estos autores evidenciaron la necesidad de realizar estudios in vitro e in vivo de la sensibilidad al benzonidazol de cepas colombianas de T. cruzi, para demostrar que las cepas circulantes en los países andinos, tienen una mayor sensibilidad al tratamiento con benzonidazol que las cepas de los países del Cono Sur y Bolivia. Estos estudios son de gran importancia para el tratamiento de esta parasitosis, porque permitirán en un futuro orientar a los organismos de salud sobre las dosis que se deben utilizar en pacientes o regiones determinadas, para evitar el surgimiento de la resistencia.

En el presente trabajo se evaluó la sensibilidad al benzonidazol de 33 cepas colombianas aisladas de diferentes orígenes biológicos y geográficos, y se hicieron algunas correlaciones con diferentes parámetros biológicos y ecoepidemiológicos. Los resultados mostraron la gran variabilidad en la sensibilidad al benzonidazol de las cepas colombianas, principalmente, según su origen biológico y carácter endémico de la zona de origen de los aislamientos. Además, la correlación de la sensibilidad al medicamento con parámetros 
como zonas endémicas, tipo de vector, hábitat del vector y año de aislamiento de la cepa, apoya la existencia de cepas naturalmente resistentes circulando en el país, resultados que son de gran importancia para la epidemiología y tratamiento de la enfermedad de Chagas en Colombia.

\section{Materiales y métodos}

\section{Reactivos}

El benzonidazol fue purificado por extracción orgánica a partir de tabletas Rochagan ${ }^{\mathrm{TM}}$ (Roche, Brasil). A partir del compuesto purificado, se preparó una solución madre a una concentración final de 10 $\mathrm{mM}$, disuelta en dimetilsulfóxido (DMSO) al $100 \%$.

\section{Material biológico}

En este estudio, se analizaron 33 cepas colombianas de $T$. cruzi clasificadas como Tcl aisladas de diferentes orígenes biológicos y de distintas áreas geográficas de Colombia con bajo, mediano y alto riesgo de contraer la infección (26) (cuadro 1). Además, como cepas de referencia se incluyeron las cepas Colombiana (Tcl) y Noel (Tcll), aisladas de humanos de Colombia y Brasil, respectivamente, y consideradas naturalmente resistentes (8). Los cultivos de epimastigotes se mantuvieron a $28{ }^{\circ} \mathrm{C}$ en medio de infusión de hígado-triptosa (LIT) (Liver Infusion Tryptose) con suplemento de suero bovino fetal (SBF) al 10 $\%$ y se subcultivaron cada siete días en fase de crecimiento exponencial.

\section{Sensibilidad al benzonidazol}

La sensibilidad al benzonidazol para las 33 cepas colombianas y las dos de referencia se hizo mediante el micrométodo enzimático de bromuro de (3-(4,5-dimetiltiazol-2-il)-2,5-difenil tetrazolio (MTT) (12). Para esto se utilizaron parásitos en fase exponencial de crecimiento, a una concentración de $1 \times 10^{7}$ parásitos $/ \mathrm{ml}$, cultivados en medio líquido LIT durante 72 horas a $28{ }^{\circ} \mathrm{C}$, con exposición a siete concentraciones diferentes de benzonidazol. Como control se utilizaron parásitos cultivados en ausencia de los medicamentos, pero mantenidos en las mismas condiciones.

Cada concentración de los medicamentos, al igual que los controles sin tratamiento, se evaluó por triplicado y en dos experimentos independientes.

Luego de las 72 horas de incubación, el efecto del benzonidazol en los parásitos se determinó midiendo la actividad de la deshidrogenasa mitocondrial, adicionando $10 \mu \mathrm{l}$ por pozo de MTT- metosulfato de fenazina (PMS) e incubando a $28^{\circ} \mathrm{C}$ durante 90 minutos. Para detener la reacción y disolver los cristales formados, se agregaron $100 \mu \mathrm{l}$ por pozo de una solución de isopropanol al $50 \%$ y de dodecilsulfato sódico (SDS) al $10 \%$. La producción de formazán se midió a $595 \mathrm{~nm}$ en un lector para ELISA (Enzyme-Linked ImmunoSorbent Assay) (Bio-Rad) y los datos se analizaron con el programa GraphPad Prism ${ }^{\mathrm{TM}}$, versión 5,01 para hallar la concentración inhibitoria $50\left(\mathrm{Cl}_{50}\right)$ al benzonidazol, mediante un análisis de regresión no lineal.

Con los datos obtenidos de $\mathrm{Cl}_{50}$ para cada uno de los ensayos, se calculó el coeficiente de variación, para estimar el grado de dispersión de las $\mathrm{Cl}_{50}$ obtenidas de dos ensayos independientes. Cuanto mayor era el coeficiente de variación, mayor era la dispersión y menor la representatividad de la media. Por lo tanto, cuando dicho valor fue superior al $20 \%$ se hizo un tercer ensayo (27).

\section{Análisis de los datos}

Los resultados obtenidos de las $\mathrm{Cl}_{50}$ para el benzonidazol de las 33 cepas colombianas, se sometieron a un análisis estadístico descriptivo usando los percentiles 25 y 75 , y se construyó un dendrograma por un análisis de conglomerados usando el método de Ward, lo que permitió clasificar las cepas según sus $\mathrm{Cl}_{50}$ en tres categorías: sensibles, parcialmente resistentes y resistentes.

Además, se hicieron diferentes comparaciones de las sensibilidades al benzonidazol con el origen biológico, especie del vector, hábitats de los vectores, área de riesgo de transmisión del parásito y año de aislamiento de las cepas. Para esto, luego de examinar los supuestos de normalidad para los datos, se utilizaron las pruebas no paramétricas de Mann-Whitney o Kruskal-Wallis, según las comparaciones hechas, con una significancia estadística de $95 \%$, en el programa estadístico GraphPad Prism ${ }^{\mathrm{TM}}$, versión 5,01. Finalmente, los rangos establecidos se compararon con los valores obtenidos para las dos cepas de referencia.

\section{Resultados}

\section{Sensibilidad de las cepas colombianas al benzonidazol}

El análisis de las $\mathrm{Cl}_{50}$ al benzonidazol de las 33 cepas colombianas mostró un amplio rango de concentraciones, que variaban desde 1,23 $\mu \mathrm{M}$ para la cepa FCh hasta $41,61 \mu \mathrm{M}$ para la cepa SO9 (cuadro 1). 
Cuadro 1. Cepas de Trypanosoma cruzi utilizadas en este estudio, con sus respectivos orígenes biológico y geográfico, año de aislamiento y sensibilidad al benzonidazol

\begin{tabular}{|c|c|c|c|c|c|c|}
\hline Cepa & Origen biológico & Origen geográfico & $\begin{array}{c}\text { Zona de } \\
\text { riego }\end{array}$ & $\begin{array}{c}\text { Año de } \\
\text { aislamiento }\end{array}$ & $\begin{array}{l}\mathrm{Cl}_{50} \\
(\mu \mathrm{M})\end{array}$ & Categoría \\
\hline FCh & Homo sapiens & Norte de Santander & Alta & 1992 & 1,23 & \multirow{12}{*}{$\begin{array}{c}\text { Sensibles } \\
36 \%\end{array}$} \\
\hline CAS18 & Didelphis marsupialis & Casanare & Alta & 2002 & 3,90 & \\
\hline YLY & H. sapiens & Putumayo & Media/baja & 2004 & 4,38 & \\
\hline MG8 & Triatoma dimidiata & Magdalena & Media/baja & 2003 & 4,56 & \\
\hline$C G$ & H. sapiens & Caquetá & Alta & 2003 & 4,61 & \\
\hline $\mathrm{HA}$ & H. sapiens & Casanare & Alta & 1997 & 4,66 & \\
\hline $\mathrm{AF} 1$ & Panstrongylus geniculatus & Antioquia & Media/baja & 1993 & 4,69 & \\
\hline MR & H. sapiens & Cesar & Media/baja & 2004 & 4,71 & \\
\hline MG & H. sapiens & Arauca & Alta & 2004 & 4,90 & \\
\hline JEM & H. sapiens & Putumayo & Media/baja & 2005 & 5,19 & \\
\hline SPR & H. sapiens & Casanare & Alta & 1982 & 5,32 & \\
\hline GAL61 & Rattus rattus & Sucre & Media/baja & 1991 & 5,85 & \\
\hline SP & H. sapiens & Casanare & Alta & 2005 & 6,41 & \multirow{16}{*}{$\begin{array}{c}\text { Parcialmente } \\
\text { resistentes } \\
48 \%\end{array}$} \\
\hline $\mathrm{AC} 17$ & Rhodnius pallescens & Chocó & Media/baja & 1999 & 6,53 & \\
\hline $\mathrm{JL}$ & H. sapiens & Norte de Santander & Alta & 1992 & 7,22 & \\
\hline GAL52 & D. marsupialis & Sucre & Media/baja & 1991 & 9,07 & \\
\hline CAS15 & R. prolixus & Casanare & Alta & 2000 & 9,93 & \\
\hline AC29 & R. pallescens & Chocó & Media/baja & 1999 & 11,10 & \\
\hline STP3.3 & R. prolixus & Tolima & Alta & 1991 & 11,26 & \\
\hline W3534 & H. sapiens & Sucre & Media/baja & 1998 & 14,01 & \\
\hline MG10 & T. dimidiata & Magdalena & Media/baja & 2003 & 14,91 & \\
\hline SN6 & R. prolixus & Guajira & Media/baja & 2003 & 16,94 & \\
\hline LB53 & T. dimidiata & Sucre & Media/baja & 1999 & 17,02 & \\
\hline OV1 & P. geniculatus & Sucre & Media/baja & 1998 & 17,44 & \\
\hline AMP07 & P. geniculatus & Antioquia & Media/baja & 1997 & 17,56 & \\
\hline B138 & T. dimidiata & Córdoba & Media/baja & 1999 & 17,60 & \\
\hline B114 & T. dimidiata & Córdoba & Media/baja & 1999 & 18,67 & \\
\hline B51 & R. pallescens & Córdoba & Media/baja & 1999 & 20,56 & \\
\hline OV17 & P. geniculatus & Sucre & Media/baja & 1998 & 22,17 & \multirow{5}{*}{$\begin{array}{c}\text { Resistentes } \\
16 \%\end{array}$} \\
\hline SN5 & R. prolixus & Guajira & Media/baja & 2003 & 24,17 & \\
\hline DA & H. sapiens & Boyacá & Alta & 2001 & 32,75 & \\
\hline SN3 & R. prolixus & Guajira & Media/baja & 2003 & 34,62 & \\
\hline SO9 & R. pallescens & Sucre & Media/baja & 1995 & 41,61 & \\
\hline
\end{tabular}

El análisis descriptivo de las $\mathrm{Cl}_{50}$, mediante la obtención de los percentiles 25 y 75, clasificó las cepas de acuerdo con su sensibilidad al benzonidazol, en tres grupos: para $25 \%$ de las cepas los valores de $\mathrm{Cl}_{50}$ fueron menores de 4,8 $\mu \mathrm{M}$; para el $50 \%$ de las cepas, los valores de $\mathrm{Cl}_{50}$ estuvieron entre 4,8 y $17,58 \mu \mathrm{M}$, y para el $25 \%$ restante, por encima de $17,58 \mu \mathrm{M}$.

Cuando se construyó el dendrograma basado en las $\mathrm{Cl}_{50}$ utilizando el algoritmo de Ward, se corroboró la presencia de tres grupos principales observados mediante el análisis de percentiles (figura 1). En el grupo más externo se ubicaron las cepas con las $\mathrm{Cl}_{50}$ más altas, con valores entre 32,75 y $41,61 \mu \mathrm{M}$, aisladas de diferentes orígenes biológicos y geográficos. En el segundo grupo se ubicaron las cepas aisladas esencialmente de vectores con $\mathrm{Cl}_{50}$ al benzonidazol desde 9,07 hasta $24,17 \mu \mathrm{M}$, mientras que en el tercer grupo se agruparon aquellas cepas aisladas principalmente de humanos y otros reservorios con sensibilidades al benzonidazol entre 1,23 y 7,22 $\mu \mathrm{M}$ (figura 1).

Con los rangos obtenidos mediante los dos análisis se clasificaron las cepas en tres categorías principales, utilizando un promedio de los límites de cada uno de los grupos obtenidos, de la siguiente manera: aquellas cepas que presentaron valores menores de $6 \mu \mathrm{M}$ se consideraron sensibles, entre 6 y $21 \mu \mathrm{M}$, parcialmente resistentes y, por encima de $21 \mu \mathrm{M}$, resistentes al benzonidazol. Estos datos fueron validados con los valores obtenidos de $\mathrm{Cl}_{50}$ para las cepas Colombiana y Noel de 30 y $22 \mu \mathrm{M}$, respectivamente, que en estudios previos in vivo fueron clasificadas como naturalmente resistentes (8). Teniendo en cuenta esta agrupación, se obtuvo que $36 \%$ de las cepas analizadas eran sensibles al benzonidazol, el $48 \%$, parcialmente resistentes y, el $16 \%$ resistentes. Es importante resaltar que el 


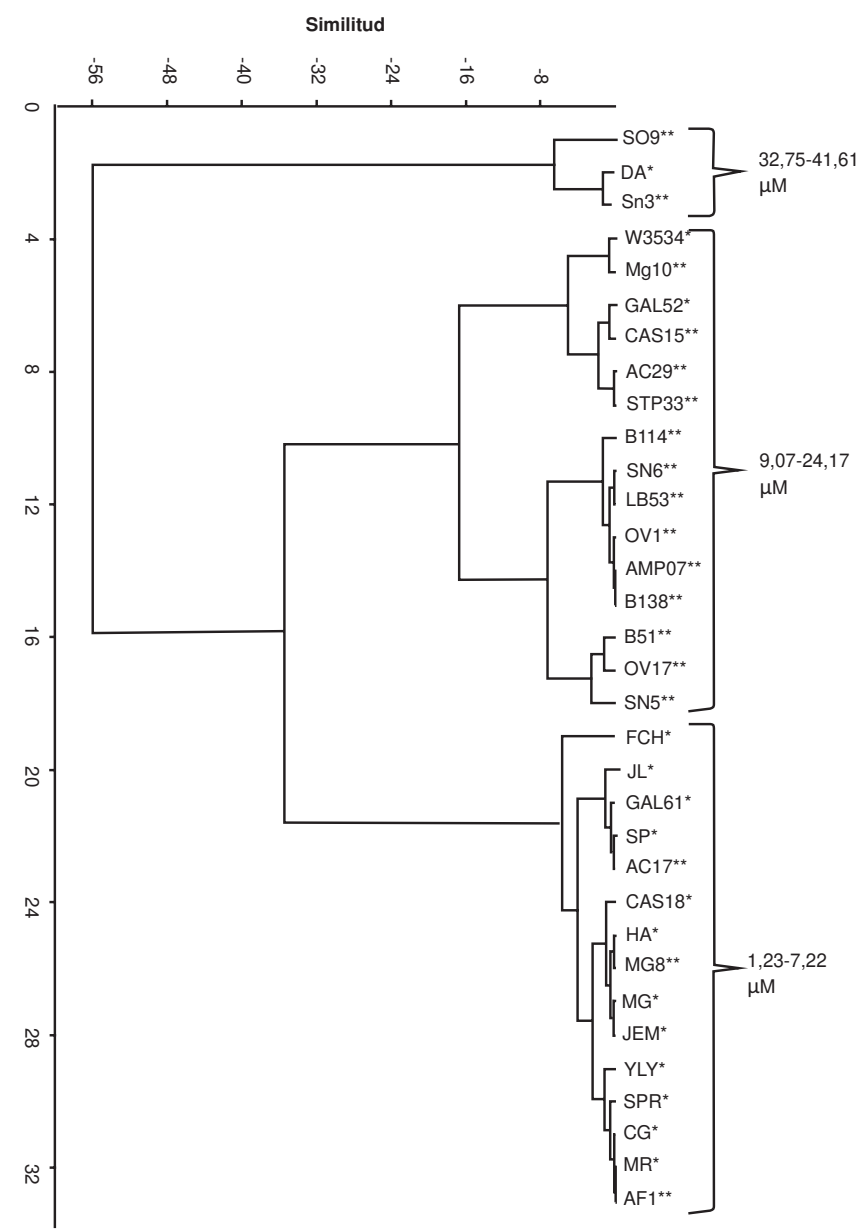

Figura 1. Análisis de conglomerados mediante el algoritmo de Ward, basados en la $\mathrm{Cl}_{50}$ para benzonidazol de epimastigotes de 33 cepas de Trypanosoma cruzi aisladas de vectores $\left({ }^{* *}\right)$, humanos $\left(^{*}\right)$ y mamíferos $\left({ }^{*}\right)$. Se indican los rangos de sensibilidad al benzonidazol $(\mu \mathrm{M})$ para cada uno de los grupos.

$67 \%, 19 \%$ y $17 \%$ de las cepas pertenecientes a cada uno de los grupos, respectivamente, correspondieron a cepas aisladas de humano (cuadro 1).

Relación entre la sensibilidad al benzonidazol de las cepas colombianas y diferentes parámetros biológicos y eco-epidemiológicos

Con el fin de establecer si la $\mathrm{Cl}_{50}$ al benzonidazol de las cepas se correlacionaba con los diferentes parámetros biológicos y eco-epidemiológicos, se hicieron diferentes comparaciones.

En un primer análisis se trató de establecer relaciones entre la sensibilidad al benzonidazol y los diferentes orígenes biológicos de las cepas (aisladas de vectores y reservorios). Los resultados obtenidos mostraron que variaba significativamente cuando las cepas se habían aislado de vectores $\left(\mathrm{Cl}_{50}\right.$ promedio de $\left.17,30 \mu \mathrm{M}\right)$, en contraste con aquellas aisladas de reservorios principalmente humanos $\left(\mathrm{Cl}_{50}\right.$ promedio de 7,61 $\left.\mu \mathrm{M}\right)$ (figura $\left.2 \mathrm{~A}\right)$.
En un segundo análisis se compararon las $\mathrm{Cl}_{50}$ con la especie del vector de la cual fue aislada la cepa, lo cual no evidenció diferencias significativas, con valores promedio de sensibilidad de 14,55, $15,47,19,38$ y $19,95 \mu \mathrm{M}$ para las cepas aisladas de Triatoma dimidiata, Panstrongylus geniculatus, Rhodnius prolixus y $R$. pallescens, respectivamente (figura 2B).

Además, el hábitat del insecto (domiciliado, silvestre) tampoco mostró una influencia en la sensibilidad que tienen las cepas al benzonidazol, encontrándose una $\mathrm{Cl}_{50}$ promedio de 20,56 $\mu \mathrm{M}$ para las cepas aisladas de vectores domiciliados y de $17,23 \mu \mathrm{M}$ para las provenientes de vectores silvestres (figura $2 \mathrm{C}$ ).

Por otro lado, el año de aislamiento de la cepa (antes o después del 2002, cuando se empezó a suministrar el tratamiento en el país) no mostró diferencias entre los dos grupos establecidos y se 
A

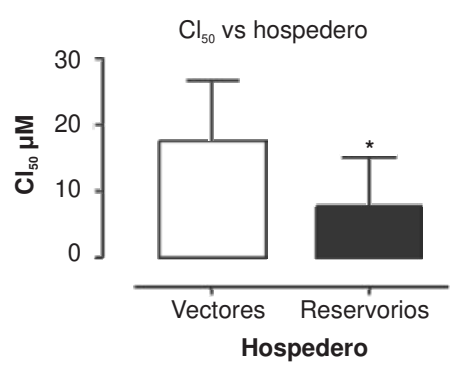

B

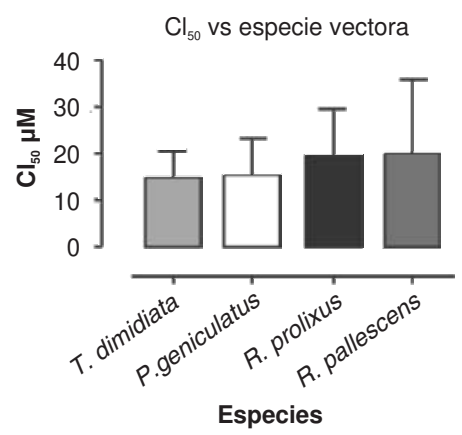

C

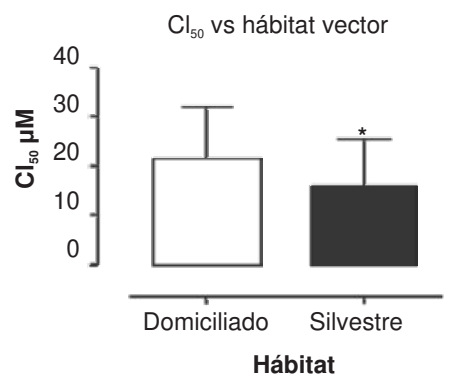

D

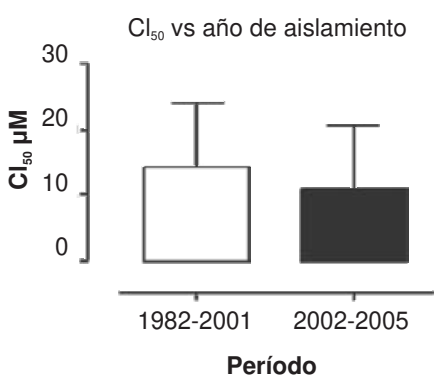

E

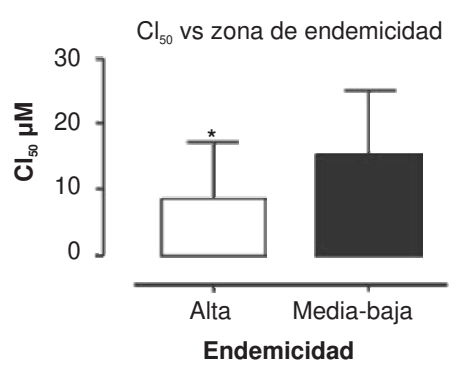

Figura 2. Correlación de la $\mathrm{Cl}_{50}$ al benzonidazol y diferentes parámetros biológicos y eco-epidemiológicos, como tipo de huésped $(A)$, especie vector $(B)$, hábitat del vector $(C)$, año de aislamiento de la cepa $(D)$ y zona de riesgo de transmisión $(E)$

encontró que las cepas aisladas entre 1985 y 2001 presentaron valores promedio de sensibilidad de $14,11 \mu \mathrm{M}$ y, las aisladas desde 2002 a 2005, de $10,78 \mu \mathrm{M}$ (figura 2D).

Finalmente, se encontraron diferencias significativas entre la zona de aislamiento de las cepas y sus respectivas $\mathrm{Cl}_{50}$; se encontró que las cepas provenientes de zonas de alta endemia poseen mayor sensibilidad al benzonidazo,I con valores promedio de $8,38 \mu \mathrm{M}$, que las cepas aisladas de zonas de mediano y bajo riesgo de transmisión, que poseen una $\mathrm{Cl}_{50}$ promedio de $15,15 \mu \mathrm{M}$ (figura $2 \mathrm{E})$.

\section{Discusión}

La enfermedad de Chagas continúa siendo un problema importante de salud pública en varios países americanos, a pesar de las diversas estrategias de control vectorial emprendidas en el continente $(1-3,28)$. El panorama se complica aún más por la ineficiente quimioterapia que existe para esta enfermedad, ya que el benzonidazol y el nifurtimox son efectivos sólo bajo ciertas circunstancias, tienen diversos efectos secundarios $(6,29)$ y pueden generar resistencia en $T$. cruzi (13$15)$, además de la existencia de cepas naturalmente resistentes a dichos medicamentos (8).
En los últimos años, diversos estudios se han enfocado en la búsqueda y evaluación de nuevas estrategias quimioterapéuticas para tratar esta enfermedad (29-38). Sin embargo, luego de más de 100 años de descubrirse la enfermedad y de más de 40 de implementarse el tratamiento, las únicas alternativas existentes son los medicamentos mencionados anteriormente (38).

En este sentido, hoy en día se están haciendo muchos esfuerzos por entender los mecanismos de acción del benzonidazol y el nifurtimox, con el fin de mejorar el uso de estos medicamentos $(7,12,13,39,40)$. Si bien es cierto que se han producido algunos avances en este sentido $(7,12,13,41,42)$, en la actualidad aún no se han podido esclarecer parámetros tales como cuáles son las concentraciones del medicamento para considerar las cepas como resistentes y cuál es la relación entre los mecanismos de resistencia natural y la inducida. Además, existen pocos reportes sobre la correlación de la sensibilidad al benzonidazol con parámetros biológicos y genéticos del parásito, como la diversidad filogenética y la sensibilidad natural e inducida, entre otros, y los resultados obtenidos de estos estudios son controversiales (22,43-46).

En el presente trabajo se analizó la sensibilidad al benzonidazol de 33 cepas colombianas aisladas 
de diferentes orígenes biológicos y geográficos, con el fin de esclarecer algunos parámetros como cuál es la sensibilidad de las cepas que circulan en Colombia, si existen algunas características biológicas y eco-epidemiológicas que afecten dicha sensibilidad en estas cepas y si en el país circulan cepas naturalmente resistentes.

Los resultados obtenidos indicaron una gran heterogeneidad en la sensibilidad al benzonidazol en las cepas colombianas, con $\mathrm{Cl}_{50}$ desde $1,23 \mu \mathrm{M}$ hasta 41,61 $\mu \mathrm{M}$. Este resultado no es sorprendente, teniendo en cuenta otros trabajos similares con cepas de Colombia, que mostraron rangos de sensibilidad entre $7,33 \mu \mathrm{M}$ y $27,30 \mu \mathrm{M}$ (47). Las $\mathrm{Cl}_{50}$ obtenidas permitieron proponer una clasificación en tres grupos según la sensibilidad o resistencia al benzonidazol, que evidencia la presencia de, al menos, $16 \%$ de cepas con resistencia natural al benzonidazol en Colombia.

En estudios anteriores, luego de estudiar el porcentaje de curación en ratones, Filardi y Brener determinaron la presencia de cepas de $T$. cruzi sensibles, parcialmente resistentes y resistentes al benzonidazol y nifurtimox, tomando como nivel arbitrario el $50 \%$ de curación en ratones (8). Sin embargo, aparte de los estudios llevados a cabo mediante inducción in vitro e in vivo por presiones con los medicamentos $(12-15,48)$, no existen otros estudios que establezcan cuáles son las concentraciones para considerar una cepa como resistente. Aunque los estudios in vivo, como los de Filardi y Brener, resultan como una mejor aproximación en la definición de estas categorías biológicas, la metodología por ellos utilizada es dispendiosa como método de tamización (8).

Por lo tanto, el uso del micrométodo enzimático del MTT resulta ser una estrategia de rápida aplicación para orientar los tratamientos en los pacientes con la enfermedad de Chagas. Para validar nuestros resultados se incluyeron dos cepas consideradas naturalmente resistentes por sus porcentajes de curación en ratones (8). Al determinar la $\mathrm{Cl}_{50}$ de estas cepas, se obtuvieron valores que están dentro de los rangos definidos para las cepas resistentes. Además, la búsqueda de reportes de $\mathrm{Cl}_{50}$ in vitro para estas y otras cepas clasificadas según su sensibilidad in vivo (8), coinciden con los rangos propuestos en este estudio. Así, para la cepa $\mathrm{Y}$, considerada parcialmente resistente, se han reportado $\mathrm{Cl}_{50}$ de $16,3 \mu \mathrm{M}(49)$ y $20 \mu \mathrm{M}(47)$, mientras que para las cepas Colombiana y Yuyu, consideradas resistentes, los reportes son de 25,4 $\mu \mathrm{M}$ y $32 \mu \mathrm{M}$ (49), respectivamente, resultados que validan los rangos propuestos en este trabajo y que habían sido validados ya por otros autores (49).

En este sentido, los datos obtenidos en este trabajo indican que en Colombia circulan cepas con $\mathrm{Cl}_{50}$ de $41,61 \mu \mathrm{M}$ para el benzonidazol, por lo que se infiere que a la concentración terapéutica que se encuentra el benzonidazol en el plasma de los pacientes $(50 \mu \mathrm{M})(14)$, la mitad de los parásitos quedarían vivos, proliferando y generando una falla quimioterapéutica. Sin embargo, hay que tener en cuenta que no siempre existe correlación entre la sensibilidad in vitro y el desenlace terapéutico (49) y, por lo tanto, deben tenerse en cuenta otras características como la sensibilidad en diferentes estadios del parásito, el estado inmunológico de los pacientes y la selección o inducción de resistencia, ya que, como ha sido reportado, los aislamientos pueden duplicar su resistencia luego del tratamiento (49). Por esta razón, sugerimos que a los aislamientos de epimastigotes que estén dentro del rango de resistentes se les podría determinar otros parámetros, como la $\mathrm{Cl}_{50}$ en otros estadios y la composición clonal, y podrían evaluarse algunos marcadores moleculares que se generarán producto de los diferentes estudios que se está realizando en $T$. cruzi sobre la resistencia natural e inducida.

A pesar de que no existe una correlación completa entre sensibilidad y resultado terapéutico (49), consideramos que este resultado es relevante en la epidemiología de la enfermedad de Chagas en el país, pues poca información hay sobre la sensibilidad de las cepas en Colombia y, menos aún, sobre la existencia de cepas resistentes que circulan en el país, y los resultados encontrados en este trabajo demuestran que, aproximadamente, el $8 \%$ (una cepa resistente de 12) de las cepas aisladas de humanos podrían ser resistentes al tratamiento con el benzonidazol. En este sentido, el estudio llevado a cabo en escolares de una región de Colombia encontró que en $24 \%$ de los niños tratados no hubo conversión serológica negativa a los seis meses (24), lo que podría deberse en parte a la resistencia natural que poseen las cepas de T. cruzi, según lo encontrado en este estudio, a factores propios del huésped, al corto tiempo de seguimiento o a ambas cosas.

Por otra parte, se trató de correlacionar la sensibilidad al benzonidazol de las cepas estudiadas con diferentes características biológicas y epidemiológicas. Con respecto al origen biológico, se 
encontró que, en general, cuando las cepas han sido aisladas de insectos vectores, necesitan mayores concentraciones de benzonidazol para inhibir su crecimiento, que las aisladas de huéspedes vertebrados, principalmente humanos. Este resultado podría explicarse por la diversidad clonal que pueden tener las cepas aisladas de ambos orígenes (50). En general, en los insectos vectores circulan cepas con mayor diversidad de clones, mientras que los huéspedes actúan como filtros biológicos, seleccionando sólo unos genotipos de la población total, lo que sugiere que en vectores y huéspedes circula una cantidad de genotipos propios, que presentan características biológicas diferentes (51).

En cuanto a los otros parámetros evaluados, se observó que aquellas áreas con baja y media endemia poseen cepas que presentan, en promedio, mayor resistencia al benzonidazol. Este resultado, aunque parece contradictorio con los procesos de expresión de resistencia, pues se esperaría mayor resistencia en zonas de alta endemia donde ya se ha implementado el tratamiento, es, en realidad, sugestivo de la resistencia natural de algunas de las cepas colombianas.

La resistencia natural puede presentarse en respuesta a otros compuestos que circulan en el ambiente, lo cual altera características fenotípicas y genotípicas de las poblaciones, haciendo que los organismos puedan soportar los medicamentos que se utilizan para el tratamiento, así no haya sido a éstos hacia los cuales se dio la expresión de la característica o rasgo particular (9). En este sentido, al ser justo las cepas más resistentes, aisladas de aquellas regiones donde posiblemente no se ha suministrado el tratamiento, se infiere que las cepas colombianas de $T$. cruzi podrían poseer resistencia natural al benzonidazol que no depende del uso de los medicamentos, que pueden generar procesos de inducción de resistencia. Por lo tanto, sugerimos a los organismos responsables de los programas de control que la resistencia natural debe ser considerada en el momento de la implementación de los tratamientos, incluso en zonas donde nunca se hayan suministrado medicamentos, como las de baja y media endemia.

En cuanto al resto de los parámetros evaluados, no se observó una relación con la sensibilidad al benzonidazol y otras características, como el tipo de vector, el tipo de hábitat del vector y el año de aislamiento de la cepa. De igual manera, estos parámetros aportan evidencia de la resistencia natural al benzonidazol para T. cruzien nuestro país.
Por ejemplo, al no observarse una diferencia en la sensibilidad cuando las cepas han sido aisladas antes o después de que se empezó a suministrar el medicamento en Colombia, se corrobora la hipótesis planteada anteriormente, en que las características que confieren la resistencia fueron adquiridas a otros compuestos diferentes a los utilizados para el tratamiento de la enfermedad de Chagas, que pueden activar las mismas vías metabólicas y que confieren a la vez resistencia al benzonidazol.

Como se mencionó anteriormente, durante la expresión de resistencia a un medicamento se pueden dar varias situaciones, presentándose fenómenos de selección o adquisición de resis-tencia seguida de selección (9-11,52-55). Por ello, como la mayor diversidad clonal se presenta en los vectores, es muy posible que en estas poblaciones haya más clones con resistencia natural al benzonidazol.

En la actualidad, diversos autores han tratado de correlacionar los mecanismos de resistencia natural con los de resistencia inducida (16-21). Sin embargo, hasta el momento no se han encontrado patrones de expresión similares en cepas que presentan los dos tipos de resistencia, lo que ha llevado a concluir que diferentes mecanismos actúan en ambos tipos de resistencia. En este sentido, el reconocimiento de cepas resistentes circulando en el país, es muy importante, pues permitiría el análisis de estos fenotipos para tratar de esclarecer los mecanismos particulares de este tipo de resistencia y determinar la presencia de genes particulares involucrados con esta característica y, por lo tanto, de biomarcadores de resistencia natural.

Finalmente, es importante resaltar que a la fecha no se había demostrado la presencia de cepas resistentes en Colombia y los pocos estudios con pacientes apuntaban a que las cepas circulantes en el país tenían mayor sensibilidad al benzonidazol, que las de otras regiones geográficas del continente. En este trabajo se evidencia la presencia de resistencia natural a este medicamento, aspecto que debe ser tenido en cuenta cuando se estén definiendo políticas de tratamiento, que puedan orientar a los investigadores y personal de salud para determinar si las cepas aisladas de pacientes responderían adecuadamente o no al tratamiento.

\section{Conflicto de intereses}

Todos los autores de este trabajo declaramos que no incurrimos en conflicto de intereses alguno durante la realización del estudio. 


\section{Financiación}

Este trabajo fue financiado por el proyecto Sostenibilidad 2010-2011, CODI, Universidad de Antioquia.

\section{Referencias}

1. Pinto-Dias J, Schofield C. Social and medical aspects: Morbidity and mortality in general population. En: Telleria $\mathrm{J}$, Tibayrenc M, editors. American trypanosomiasis Chagas disease. One hundred years of research. First edition. London: Elsevier; 2010. p. 45-54.

2. Patterson J, Guhl F. Geographical distribution of Chagas disease. En: Telleria J, Tibayrenc M, editors. American trypanosomiasis Chagas disease. One hundred years of research. First edition. London: Elsevier; 2010. p. 83-114.

3. Moncayo A, Silveira A. Current trends and future prospects for control of Chagas disease. En: Telleria J, Tibayrenc M, editors. American trypanosomiasis Chagas disease. One hundred years of research. First edition. London: Elsevier; 2010. p. 55-82.

4. Moncayo A. Chagas disease: Current epidemiological trends after the interruption of vectorial and transfusional transmission in the Southern Cone countries. Mem Inst Oswaldo Cruz. 2003;98:577-91.

5. López-Antuñano FJ. Quimioterapia de las infecciones producidas por Trypanosoma cruzi. Salud Pública Mex. 1997;39:463-71.

6. Rodrigues J, Castro S. A critical review on Chagas disease chemotherapy. Mem Inst Oswaldo Cruz. 2002;97:3-24.

7. Wilkinson SR, Kelly JM. Trypanocidal drugs: Mechanisms, resistance and new targets. Expert Rev Mol Med. 2009;11:124.

8. Filardi LS, Brener Z. Susceptibility and natural resistance of Trypanosoma cruzi strains to drugs used clinically in Chagas disease. Trans R Soc Trop Med Hyg. 1987;81:755-9.

9. Hayes JD, Wolf CR. Molecular mechanisms of drug resistance. Biochem J. 1990;272:281-95.

10. Ouellette M. Biochemical and molecular mechanisms of drug resistance in parasites. Trop Med Int Health. $2001 ; 6: 874-82$.

11. Singh N. Drug resistance mechanisms in clinical isolates of Leishmania donovani. Indian J Med Res. 2006;123:41122.

12. Mejía-Jaramillo AM, Fernández GJ, Palacio L, Triana 0. Gene expression study using real-time PCR identifies an NTR gene as a major marker of resistance to beznidazole in Trypanosoma cruzi. Parasit Vectors. 2011;4:1-12.

13. Wilkinson SR, Taylor MC, Horn D, Kelly JM, Cheeseman I. A mechanism for cross-resistance to nifurtimox and benznidazole in trypanosomes. Proc Natl Acad Sci USA. 2008;105:5022-7.

14. Villarreal D, Nirde P, Hide M, Barnabe C, Tibayrenc M. Differential gene expression in benznidazole-resistant Trypanosoma cruzi parasites. Antimicrob Agents Chemother. 2005;49:2701-9.

15. Nirdé P, Larroque C, Barnabé C. Drug-resistant epimastigotes of Trypanosoma cruzi and persistence of this phenotype after differentiation into amastigotes. C R Acad Sci Ser. 1995;318:1239-44.

16. Andrade HM, Murta SM, Chapeaurouge A, Perales J, Nirdé P, Romanha AJ. Proteomic analysis of Trypanosoma cruzi resistance to benznidazole. J Proteome Res. 2008;7:2357-67.

17. Campos FM, Liarte DB, Mortara RA, Romanha AJ, Murta SM. Characterization of a gene encoding alcohol dehydrogenase in benznidazole-susceptible and -resistant populations of Trypanosoma cruzi. Acta Trop. 2009;111:56-63.

18. Murta SM, Krieger MA, Montenegro LR, Campos FF, Probst CM, Avila AR, et al. Deletion of copies of the gene encoding old yellow enzyme (TcOYE), a NAD(P) $\mathrm{H}$ flavin oxidoreductase, associates with in vitro-induced benznidazole resistance in Trypanosoma cruzi. Mol Biochem Parasitol. 2006;146:151-62.

19. Murta SM, Nogueira FB, dos Santos PF, Campos FF, Volpe C, Liarte DB, et al. Differential gene expression in Trypanosoma cruzi populations susceptible and resistant to benznidazole. Acta Trop. 2008;107:59-65.

20. Nogueira FB, Krieger MA, Nirde P, Goldenberg S, Romanha AJ, Murta SM. Increased expression of ironcontaining superoxide dismutase-A (TcFeSOD-A) enzyme in Trypanosoma cruzi population with in vitro-induced resistance to benznidazole. Acta Trop. 2006;100:119-32.

21. Nogueira FB, Ruiz JC, Robello C, Romanha AJ, Murta SM. Molecular characterization of cytosolic and mitochondrial tryparedoxin peroxidase in Trypanosoma cruzi populations susceptible and resistant to benznidazole. Parasitol Res. 2009;104:835-44.

22. Villarreal D, Barnabe C, Sereno D, Tibayrenc M. Lack of correlation between in vitro susceptibility to benznidazole and phylogenetic diversity of Trypanosoma cruzi, the agent of Chagas disease. Exp Parasitol. 2004;108:24-31.

23. Murta SM, Gazzinelli RT, Brener Z, Romanha AJ. Molecular characterization of susceptible and naturally resistant strains of Trypanosoma cruzi to benznidazole and nifurtimox. Mol Biochem Parasitol. 1998;93:203-14.

24. Guhl F, Nicholls R, Montoya R, Rosas F, Velasco V, Mora $\mathbf{E}$, et al. Rapid negativization of serology after treatment with benznidazole for Chagas disease in a group of Colombian schoolchildren. Proceedings of the IX European Multicolloquim of Parasitology. Valencia: Medimond International Proceedings; 2004. p. 107-14.

25. Padilla J. Situación de la enfermedad de Chagas en Colombia. Primer Taller Internacional Sobre Control de la Enfermedad de Chagas, Curso de diagnóstico, manejo y tratamiento de la enfermedad de Chagas, VI Reunión de la Iniciativa Andina para el Control de la Enfermedad de Chagas. Bogotá: CIMPAT; 2005. p. 19-22.

26. Guhl F, Angulo V, Restrepo M, Nicholls S, Montoya R. Estado del arte de la enfermedad de Chagas en Colombia y estrategias de control. Biomédica. 2003;23:31-3.

27. Reed GF, Lynn F, Meade BD. Use of coefficient of variation in assessing variability of quantitative assays. Clin Diagn Lab Immunol. 2002;9:1235-9.

28. Guhl F,Lazdins-Helds J. Grupo de trabajo científico sobre la enfermedad de Chagas. Buenos Aires: Organización Mundial de la Salud; 2007. 
29. Urbina JA, Docampo R. Specific chemotherapy of Chagas disease: Controversies and advances. Trends Parasitol. 2003;19:495-501.

30. Buckner FS, Navabi N. Advances in Chagas disease drug development: 2009-2010. Curr Opin Infect Dis. 2010; 23:609-16.

31. Boiani M, Boiani L, Merlino A, Hernández $P$, Chidichimo A, Cazzulo JJ, et al. Second generation of $2 \mathrm{H}$-benzimidazole 1,3-dioxide derivatives as anti-trypanosomatid agents: Synthesis, biological evaluation, and mode of action studies. Eur J Med Chem. 2009;44:4426-33.

32. Boiani M, Piacenza L, Hernández P, Boiani L, Cerecetto $\mathbf{H}$, González $\mathbf{M}$, et al. Mode of action of nifurtimox and $\mathrm{N}$-oxide-containing heterocycles against Trypanosoma cruzi: Is oxidative stress involved? Biochem Pharmacol. 2010;79:1736-45.

33. Castro D, Boiani L, Benítez D, Hernández P, Merlino A, Gil C, et al. Anti-trypanosomatid benzofuroxans and deoxygenated analogues: Synthesis using polymersupported triphenylphosphine, biological evaluation and mechanism of action studies. Eur $\mathrm{J}$ Med Chem. 2009;44:5055-65.

34. Gerpe A, Álvarez G, Benítez D, Boiani L, Quiroga M, Hernández $P$, et al. 5-Nitrofuranes and 5-nitrothiophenes with anti-Trypanosoma cruziactivity and ability to accumulate squalene. Bioorg Med Chem. 2009;17:7500-9.

35. Gerpe A, Boiani L, Hernández P, Sortino M, Zacchino S, González M, et al. Naftifine-analogues as anti-Trypanosoma cruzi agents. Eur J Med Chem. 2010;45:2154-64.

36. Pagano M, Demoro B, Toloza J, Boiani L, González $\mathbf{M}$, Cerecetto $\mathbf{H}$, et al. Effect of ruthenium complexation on trypanocidal activity of 5-nitrofuryl containing thiosemicarbazones. Eur J Med Chem. 2009;44:4937-43.

37. Rodríguez J, Arán VJ, Boiani L, Olea-Azar C, Lavaggi ML, González M, et al. New potent 5-nitroindazole derivatives as inhibitors of Trypanosoma cruzi growth: Synthesis, biological evaluation, and mechanism of action studies. Bioorg Med Chem. 2009;17:8186-96.

38. Urbina JA. Specific chemotherapy of Chagas disease: Relevance, current limitations and new approaches. Acta Trop. 2010;115:55-68.

39. Maya JD, Cassels BK, Iturriaga-Vásquez P, Ferreira J, Faundez M, Galanti N, et al. Mode of action of natural and synthetic drugs against Trypanosoma cruzi and their interaction with the mammalian host. Comp Biochem Physiol A Mol Integr Physiol. 2007;146:601-20.

40. Maya JD, Bollo S, Núñez-Vergara LJ, Squella JA, Repetto Y, Morello A, et al. Trypanosoma cruzi: Effect and mode of action of nitroimidazole and nitrofuran derivatives. Biochem Pharmacol. 2003;65:999-1006.

41. Hall BS, Wu X, Hu L, Wilkinson SR. Exploiting the drugactivating properties of a novel trypanosomal nitroreductase. Antimicrob Agents Chemother. 2010;54:1193-9.

42. Hall BS, Bot C, Wilkinson SR. Nifurtimox activation by trypanosomal type I nitroreductases generates cytotoxic nitrile metabolites. J Biol Chem. 2011;286:13088-95.
43. Toledo MJ, Bahia MT, Carneiro CM, Martins-Filho OA, Tibayrenc M, Barnabe C, et al. Chemotherapy with benznidazole and itraconazole for mice infected with different Trypanosoma cruzi clonal genotypes. Antimicrob Agents Chemother. 2003;47:223-30.

44. Veloso VM, Carneiro CM, Toledo MJ, Lana M, Chiari E, Tafuri WL, et al. Variation in susceptibility to benznidazole in isolates derived from Trypanosoma cruzi parental strains. Mem Inst Oswaldo Cruz. 2001;96:1005-11.

45. Camandaroba EL, Reis EA, Goncalves MS, Reis MG, Andrade SG. Trypanosoma cruzi: Susceptibility to chemotherapy with benznidazole of clones isolated from the highly resistant Colombian strain. Rev Soc Bras Med Trop. 2003;36:201-9.

46. Triana O, Mejía A, Zapata C, Arboleda A, Dib J. Caracterización genética y sensibilidad al benzonidazole de cepas colombianas de Trypanosoma cruzi. Biomédica. $2005 ; 25: 82-5$

47. Luna KP, Hernández IP, Rueda CM, Zorro MM, Croft SL, Escobar P. In vitrosusceptibility of Trypanosoma cruzistrains from Santander, Colombia, to hexadecylphosphocholine (miltefosine), nifurtimox and benznidazole. Biomédica. 2009;29:448-55

48. Murta SM, Romanha AJ. In vivo selection of a population of Trypanosoma cruzi and clones resistant to benznidazole. Parasitol. 1998;116:165-71.

49. Moreno M, D'ávila DA, Silva MN, Galvão LM, Macedo AM, Chiari E, et al. Trypanosoma cruzi benznidazole susceptibility in vitro does not predict the therapeutic outcome of human Chagas disease. Mem Inst Oswaldo Cruz. 2010;105:918-24.

50. Tibayrenc M, Ward P, Moya A, Ayala FJ. Natural populations of Trypanosoma cruzi, the agent of Chagas disease, have a complex multiclonal structure. Proc Natl Acad Sci USA. 1986;83:115-9.

51. Macedo AM, Machado CR, Oliveira RP, Pena SD. Trypanosoma cruzi: genetic structure of populations and relevance of genetic variability to the pathogenesis of Chagas disease. Mem Inst Oswaldo Cruz. 2004;99:1-12.

52. Borst $\mathbf{P}$, Oullette $\mathbf{M}$. New mechanism of drug resistance in parasitic protozoa. Annu Rev Microbiol. 1995;49:427-60.

53. El Fadili K, Messier N, Leprohon P, Roy G, Guimond C, Trudel N, et al. Role of the ABC transporter MRPA (PGPA) in antimony resistance in Leishmania infantum axenic and intracellular amastigotes. Antimicrob Agents Chemother. 2005;49:1988-93.

54. Ouellette M, Legare D, Papadopoulou B. Multidrug resistance and $A B C$ transporters in parasitic protozoa. J Mol Microbiol Biotechnol. 2001;3:201-6.

55. Singh AK, Papadopoulou B, Ouellette M. Gene amplification in amphotericin B-resistant Leishmania tarentolae. Exp Parasitol. 2001;99:141-7. 